\title{
Synthesis of Some New Thiazole Derivatives and Their Biological Activity Evaluation
}

\author{
Leyla Yurttaş, ${ }^{1}$ Yusuf Özkay, ${ }^{1}$ Hülya Karaca Gençer, ${ }^{2}$ and Ulviye Acar ${ }^{1}$ \\ ${ }^{1}$ Department of Pharmaceutical Chemistry, Faculty of Pharmacy, Anadolu University, Eskişehir, Turkey \\ ${ }^{2}$ Department of Pharmaceutical Microbiology, Faculty of Pharmacy, Anadolu University, Eskişehir, Turkey \\ Correspondence should be addressed to Leyla Yurttaş; lyurttas@anadolu.edu.tr
}

Received 6 April 2015; Accepted 12 May 2015

Academic Editor: Filomena Conforti

Copyright (c) 2015 Leyla Yurttaş et al. This is an open access article distributed under the Creative Commons Attribution License, which permits unrestricted use, distribution, and reproduction in any medium, provided the original work is properly cited.

New 2-(4-arylpiperazine-1-yl)-N-[4-(2-(4-substituted phenyl)thiazol-4-yl)phenyl]acetamide derivatives were synthesized and evaluated for their antimicrobial and anticholinesterase activities. Acetylcholinesterase inhibitory activities of the compounds were found weak contrary to expectations. It is unlikely that antifungal activity of the compounds was found significant, especially against Candida parapsilosis.

\section{Introduction}

The treatment of infectious diseases still remains a challenging task because of combination of factors including increasing number of multidrug resistant, microbial pathogens and emerging newer infectious diseases [1]. The therapeutic problem is a crucial part of hospitalized patients, immunosuppressed patients with AIDS, and those undergoing anticancer therapy or organ transplants. In spite of a large number of antibiotics and chemotherapeutics available for medical use, the emerging resistance to old and antibiotics has created a substantial need for novel classes of antimicrobial agents [2]. So, it is necessary to design new and effective molecules as antimicrobial agents.

Small-ring heterocycles including nitrogen and sulfur have been under investigation for a long time on account of their synthetic diversity and therapeutic relevance. Among the wide range of heterocycles explored to privileged candidates in drug discovery, thiazoles have been identified to play a necessary role in medical chemistry [3].

Thiazole ring is a structural fragment of natural compounds such as thiamine (vitamin B1), thiamine pyrophosphate (TPP, a coenzyme important in respiration in the Krebs cycle), epothilones, carboxylase, and the large family of macrocyclic thiopeptide antibiotics, thiostrepton and micrococcin P1 $[4,5]$. Thiazole derivatives are associated with a broad spectrum of biological properties, including anticonvulsant, antimicrobial, antituberculous, bacteriostatic activities, antiviral, antimalarial, anticancer, hypertension, inflammation, schizophrenia, HIV infections, hypnotics and more recently for the treatment of pain, as fibrinojen receptor antagonists with antithrombotic activity, as new inhibitors of bacterial DNA gyrase B [6-10]. Thiazole derivatives are also found in application in the drug development for the treatment of allergies [11].

Many currently notable drugs contain piperazine ring and amide moiety as part of their molecular structure [12]. Piperazines are also among the most important building blocks in today's drug discovery and are found in biologically active compounds across a number of different therapeutic areas such as antifungal, antibacterial, antimalarial, and antipsychotic activities [13].

In our previous study, cholinesterase activity of some thiazole-piperazine derivatives was reported [14]. Prompted from the data we have obtained before and the above literature research, we synthesized a new series of thiazolepiperazine derivatives and investigated their antimicrobial and anticholinesterase activities so as to obtain new biologically active compounds. 


\section{Experimental}

2.1. Material. Melting points $\left({ }^{\circ} \mathrm{C}\right.$, uncorrected) of the synthesized compounds were determined by using electrothermal melting point apparatus (Electrothermal, Essex, UK). ${ }^{1} \mathrm{H}$ NMR spectra were recorded with a Bruker $500 \mathrm{MHz}$ digital FT-NMR spectrometer (Bruker Bioscience, Billerica, MA, USA) and ${ }^{13} \mathrm{C}$ NMR spectra were recorded with a Bruker $125 \mathrm{MHz}$ digital FT-NMR spectrometer in DMSO$d_{6}$ solvent including TMS as an internal standard. Coupling constants $(J)$ are reported as Hertz. IR spectra were obtained in $\mathrm{KBr}$ pressed pellets via IR, Shimadzu 8400S spectrophotometer (Shimadzu, Tokyo, Japan). Mass spectra of fast atom bombardment (FAB) were obtained on a VG Quattro mass spectrometer VG Quattro Mass spectrometer (Fisons Instruments Vertriebs GmbH, Mainz, Germany). The purities of compounds were checked by TLC on silica gel 60 $\mathrm{F}_{254}$ (Merck KGaA, Darmstadt, Germany). Starting materials were either commercially available or synthesized according to the methods given in the literature.

2.2. General Procedure for the Synthesis of 2-(4-Arylpiperazine-1-yl)-N-[4-(2-(4-substituted phenyl)thiazol-4-yl)phenyllacetamide Derivatives (6a-6p). A mixture of compound 2-chloro- $N$-[4-(2-(4-substituted phenyl)-4-thiazolyl)phenyl]acetamide 5 (1.5 mmol), appropriate piperazine derivative $(1.8 \mathrm{mmol})$ and $\mathrm{K}_{2} \mathrm{CO}_{3}(1.5 \mathrm{mmol})$ in acetone, was refluxed for $2 \mathrm{~h}$. After cooling, the solvent was evaporated until dryness. The residue was treated with $25 \mathrm{~mL}$ of water. Solidified product was filtered, washed with water, and recrystallized from ethanol to give the compounds $6 \mathbf{a}-\mathbf{6 p}$.

2.2.1. 2-(4-Benzylpiperazin-1-yl)-N-[4-(2-phenylthiazol-4-yl)phenyl]acetamide (6a). Yield 70-76\%, m.p. 171-174 ${ }^{\circ} \mathrm{C}$. IR (KBr) $\nu_{\max }\left(\mathrm{cm}^{-1}\right)$ : 3281 (amide $\left.\mathrm{N}-\mathrm{H}\right), 3005$ (aromatic $\mathrm{C}-\mathrm{H}$ ), 2912 (aliphatic C-H), 1676 (amide $\mathrm{C}=\mathrm{O}), 1512-1306(\mathrm{C}=\mathrm{C}$ and $\mathrm{C}=\mathrm{N}), 1190-978(\mathrm{C}-\mathrm{O}$ and $\mathrm{C}-\mathrm{N}) .{ }^{1} \mathrm{H}-\mathrm{NMR}(125 \mathrm{MHz}$, DMSO- $\left.d_{6}, \mathrm{ppm}\right): \delta 2.45\left(4 \mathrm{H}, \mathrm{t}, J=5 \mathrm{~Hz}, \mathrm{~N}-\mathrm{CH}_{2}\right), 2.55(4 \mathrm{H}$, t, $\left.J=4.5 \mathrm{~Hz}, \mathrm{~N}-\mathrm{CH}_{2}\right), 3.15\left(2 \mathrm{H}, \mathrm{s}, \mathrm{COCH}_{2}\right), 3.49(2 \mathrm{H}, \mathrm{s}, \mathrm{C}-$ $\left.\mathrm{CH}_{2}\right), 7.25-7.35$ (5H, m, Ar-H), 7.51-7.56 (3H, m, Ar-H), 7.75 $(2 \mathrm{H}, \mathrm{d}, J=9 \mathrm{~Hz}, \mathrm{Ar}-\mathrm{H}), 7.99-8.04(4 \mathrm{H}, \mathrm{m}, \mathrm{Ar}-\mathrm{H}), 8.09(1 \mathrm{H}$, s, Ar-H), $9.82(1 \mathrm{H}, \mathrm{s}, \mathrm{N}-\mathrm{H}) .{ }^{13} \mathrm{C}-\mathrm{NMR}\left(500 \mathrm{MHz}, \mathrm{DMSO}-d_{6}\right.$, ppm): $\delta 52.45,52.80,61.81,62.04,113.44,119.49,126.17,126.53$, $126.87,128.14,128.79,129.17,129.25,130.32,133.01,138.22$, 138.58, 154.96, 166.81, 168.35. For $\mathrm{C}_{28} \mathrm{H}_{28} \mathrm{~N}_{4}$ OS calculated: (\%) C 71.76, H 6.02, N 11.96; found: (\%) C 71.71, H 6.10, N 11.91. MS: $m / z 469(\mathrm{M}+1)$.

2.2.2. 2-[4-(Pyridin-2-yl)piperazin-1-yl]-N-[4-(2-phenylthiazol-4-yl)phenyl]acetamide (6b). Yield 70-76 \%, m.p. 173$176^{\circ} \mathrm{C}$. IR $(\mathrm{KBr}) v_{\max }\left(\mathrm{cm}^{-1}\right): 3319$ (amide N-H), 3091 (aromatic C-H), 2912 (aliphatic C-H), 1676 (amide $\mathrm{C}=\mathrm{O}$ ), 1524$1309(\mathrm{C}=\mathrm{C}$ and $\mathrm{C}=\mathrm{N}), 1246-978(\mathrm{C}-\mathrm{O}$ and $\mathrm{C}-\mathrm{N}) .{ }^{1} \mathrm{H}-\mathrm{NMR}$ $\left(500 \mathrm{MHz}, \mathrm{DMSO}-d_{6}, \mathrm{ppm}\right): \delta 2.61\left(4 \mathrm{H}, \mathrm{t}, J=5 \mathrm{~Hz}, \mathrm{~N}-\mathrm{CH}_{2}\right)$, $3.23\left(2 \mathrm{H}, \mathrm{s}, \mathrm{COCH}_{2}\right), 3.76\left(4 \mathrm{H}\right.$, brs, $\left.\mathrm{N}-\mathrm{CH}_{2}\right), 6.63-6.64(1 \mathrm{H}$, $\mathrm{m}, \mathrm{Ar}-\mathrm{H}), 6.99-7.00(1 \mathrm{H}, \mathrm{m}, \mathrm{Ar}-\mathrm{H}), 7.52-7.56(4 \mathrm{H}, \mathrm{m}, \mathrm{Ar}-$ $\mathrm{H}), 7.76(2 \mathrm{H}, \mathrm{d}, J=9 \mathrm{~Hz}, \mathrm{Ar}-\mathrm{H}), 7.84-7.85(1 \mathrm{H}, \mathrm{m}, \mathrm{Ar}-\mathrm{H})$, 8.00-8.09 (5H, m, Ar-H), $9.92(1 \mathrm{H}, \mathrm{s}, \mathrm{N}-\mathrm{H})$. For $\mathrm{C}_{26} \mathrm{H}_{25} \mathrm{~N}_{5} \mathrm{OS}$ calculated: (\%) C 68.55, H 5.53, N 15.37; found: (\%) C 68.62, H 5.63, N 15.40. MS: $m / z 456(\mathrm{M}+1)$.

2.2.3. 2-[4-(Pyrimidin-2-yl)piperazin-1-yl]-N-[4-(2-phenylthiazol-4-yl)phenyl]acetamide (6c). Yield 75-78 \%, m.p. 210$216^{\circ} \mathrm{C}$. IR $(\mathrm{KBr}) v_{\max }\left(\mathrm{cm}^{-1}\right): 3253$ (amide N-H), 3053 (aromatic C-H), 2987 (aliphatic C-H), 1674 (amide C=O), 1585$1309(\mathrm{C}=\mathrm{C}$ and $\mathrm{C}=\mathrm{N}), 1228-979(\mathrm{C}-\mathrm{O}$ and $\mathrm{C}-\mathrm{N}) .{ }^{1} \mathrm{H}-\mathrm{NMR}$ (500 MHz, DMSO- $d_{6}$, ppm): $\delta 2.59\left(4 \mathrm{H}, \mathrm{t}, J=5 \mathrm{~Hz}, \mathrm{~N}-\mathrm{CH}_{2}\right)$, $3.21\left(2 \mathrm{H}, \mathrm{s}, \mathrm{COCH}_{2}\right), 3.82\left(4 \mathrm{H}, \mathrm{t}, J=4.5 \mathrm{~Hz}, \mathrm{~N}-\mathrm{CH}_{2}\right), 6.62$ $(1 \mathrm{H}, \mathrm{t}, J=4.5 \mathrm{~Hz}, \mathrm{Ar}-\mathrm{H}), 7.38(2 \mathrm{H}, \mathrm{t}, J=7.5 \mathrm{~Hz}, \mathrm{Ar}-\mathrm{H})$, $7.77(2 \mathrm{H}, \mathrm{d}, J=8 \mathrm{~Hz}, \mathrm{Ar}-\mathrm{H}), 8.00(2 \mathrm{H}, \mathrm{d}, J=8 \mathrm{~Hz}, \mathrm{Ar}-\mathrm{H})$, 8.06-8.08 (4H, m, Ar-H), 8.35 (2H, d, $J=4.5 \mathrm{~Hz}, \mathrm{Ar}-\mathrm{H}), 9.93$ $(1 \mathrm{H}, \mathrm{s}, \mathrm{N}-\mathrm{H}) .{ }^{13} \mathrm{C}-\mathrm{NMR}\left(125 \mathrm{MHz}, \mathrm{DMSO}-d_{6}, \mathrm{ppm}\right): \delta 43.22$, $52.48,61.71,110.08,113.44,119.57,126.17,126.52,129.20,129.24$, $130.31,133.02$, 138.62, 154.97, 157.89, 161.20, 166.80, 168.28. For $\mathrm{C}_{25} \mathrm{H}_{24} \mathrm{~N}_{6} \mathrm{OS}$ calculated: (\%) C 65.77, H 5.30, N 18.41; found: (\%) C 65.67, H 5.33, N 18.36. MS: $m / z 457(\mathrm{M}+1)$.

2.2.4. 2-[4-(2-Furoyl)piperazin-1-yl]-N-[4-(2-phenylthiazol4-yl)phenyl]acetamide (6d). Yield 70-72\%, m.p. $144-145^{\circ} \mathrm{C}$. IR $(\mathrm{KBr}) v_{\max }\left(\mathrm{cm}^{-1}\right): 3215$ (amide $\left.\mathrm{N}-\mathrm{H}\right), 3034$ (aromatic C$\mathrm{H}), 2975$ (aliphatic C-H), 1676 (amide $\mathrm{C}=\mathrm{O}$ ), 1625-1308 (C=C and $\mathrm{C}=\mathrm{N}), 1263-978(\mathrm{C}-\mathrm{O}$ and $\mathrm{C}-\mathrm{N}) .{ }^{1} \mathrm{H}-\mathrm{NMR}(500 \mathrm{MHz}$, DMSO- $\left.d_{6}, \mathrm{ppm}\right): \delta 2.62\left(4 \mathrm{H}, \mathrm{t}, J=4.5 \mathrm{~Hz}, \mathrm{~N}-\mathrm{CH}_{2}\right), 3.22(2 \mathrm{H}$, $\left.\mathrm{s}, \mathrm{COCH}_{2}\right), 3.75\left(4 \mathrm{H}, \mathrm{t}, J=4.5 \mathrm{~Hz}, \mathrm{~N}-\mathrm{CH}_{2}\right), 6.62-6.63(1 \mathrm{H}, \mathrm{m}$, Ar-H), $7.03(1 \mathrm{H}, \mathrm{d}, J=3.5 \mathrm{~Hz}, \operatorname{Ar}-\mathrm{H}), 7.08(2 \mathrm{H}, \mathrm{d}, J=8.5 \mathrm{~Hz}$, Ar-H), 7.79 (3H, m, Ar-H), 7.87 (1H, s, Ar-H), 7.97-8.05 (5H, m, Ar-H), $9.93(1 \mathrm{H}, \mathrm{s}, \mathrm{N}-\mathrm{H})$. For $\mathrm{C}_{26} \mathrm{H}_{24} \mathrm{~N}_{4} \mathrm{O}_{3} \mathrm{~S}$ calculated: (\%) C 66.08, H 5.12, N 11.86; found: (\%) C 68.13, H 5.17, N 11.93. MS: $m / z 473(\mathrm{M}+1)$.

2.2.5. 2-(4-Benzylpiperazin-1-yl)-N-[4-(2-(4-methoxyphenyl)thiazol-4-yl)phenyl]acetamide (6e). Yield 80-82\%, m.p. $171-175^{\circ} \mathrm{C}$. IR (KBr) $v_{\max }\left(\mathrm{cm}^{-1}\right): 3302$ (amide N-H), 3041 (aromatic C-H), 2952 (aliphatic C-H), 1653 (amide C=O), 1587-1305 $(\mathrm{C}=\mathrm{C}$ and $\mathrm{C}=\mathrm{N}), 1244-978(\mathrm{C}-\mathrm{O}$ and $\mathrm{C}-\mathrm{N}) .{ }^{1} \mathrm{H}-$ NMR (500 MHz, DMSO- $\left.d_{6}, \mathrm{ppm}\right): \delta 2.46(4 \mathrm{H}, \mathrm{t}, J=5 \mathrm{~Hz}$, $\left.\mathrm{N}-\mathrm{CH}_{2}\right), 2.56\left(4 \mathrm{H}, \mathrm{t}, J=4.5 \mathrm{~Hz}, \mathrm{~N}-\mathrm{CH}_{2}\right), 3.14(2 \mathrm{H}, \mathrm{s}$, $\left.\mathrm{COCH}_{2}\right), 3.48\left(2 \mathrm{H}, \mathrm{s}, \mathrm{C}-\mathrm{CH}_{2}\right), 3.83\left(3 \mathrm{H}, \mathrm{s}, \mathrm{OCH}_{3}\right), 7.08(2 \mathrm{H}$, $\mathrm{d}, J=9 \mathrm{~Hz}, \mathrm{Ar}-\mathrm{H}), 7.30-7.32(5 \mathrm{H}, \mathrm{m}, \mathrm{Ar}-\mathrm{H}), 7.72(2 \mathrm{H}, \mathrm{d}$, $J=8.5 \mathrm{~Hz}, \mathrm{Ar}-\mathrm{H}), 7.95-7.98(5 \mathrm{H}, \mathrm{m}, \mathrm{Ar}-\mathrm{H}), 9.82(1 \mathrm{H}, \mathrm{s}, \mathrm{N}-$ $\mathrm{H})$. For $\mathrm{C}_{29} \mathrm{H}_{30} \mathrm{~N}_{4} \mathrm{O}_{2} \mathrm{~S}$ calculated: (\%) C 69.85, H 6.06, N 11.24; found: (\%) C 69.92, H 6.14, N 11.31. MS: $m / z 499(\mathrm{M}+1)$.

2.2.6. 2-[4-(Pyridin-2-yl)piperazin-1-yl]-N-[4-(2-(4-methoxyphenyl)thiazol-4-yl)phenyl]acetamide (6f). Yield 78-80\%, m.p. $153-154^{\circ} \mathrm{C}$. IR (KBr) $v_{\max }\left(\mathrm{cm}^{-1}\right): 3317$ (amide N-H), 3014 (aromatic C-H), 2941 (aliphatic C-H), 1656 (amide $\mathrm{C}=\mathrm{O}), 1589-1303(\mathrm{C}=\mathrm{C}$ and $\mathrm{C}=\mathrm{N}), 1247-978 \quad(\mathrm{C}-\mathrm{O}$ and C-N) ${ }^{1} \mathrm{H}-\mathrm{NMR}\left(500 \mathrm{MHz}, \mathrm{DMSO}-d_{6}, \mathrm{ppm}\right): \delta 2.63(4 \mathrm{H}$, $\left.\mathrm{t}, J=5 \mathrm{~Hz}, \mathrm{~N}-\mathrm{CH}_{2}\right), 3.21\left(2 \mathrm{H}, \mathrm{s}, \mathrm{COCH}_{2}\right), 3.56(4 \mathrm{H}, \mathrm{t}$, $\left.J=4.5 \mathrm{~Hz}, \mathrm{~N}-\mathrm{CH}_{2}\right), 3.84\left(3 \mathrm{H}, \mathrm{s}, \mathrm{OCH}_{3}\right), 6.63(1 \mathrm{H}, \mathrm{t}$, $J=6 \mathrm{~Hz}, \mathrm{Ar}-\mathrm{H}), 6.83(1 \mathrm{H}, \mathrm{d}, J=8.5 \mathrm{~Hz}, \mathrm{Ar}-\mathrm{H}), 7.08(2 \mathrm{H}$, $\mathrm{d}, J=9 \mathrm{~Hz}, \mathrm{Ar}-\mathrm{H}), 7.52(1 \mathrm{H}, \mathrm{t}, J=5 \mathrm{~Hz}, \mathrm{Ar}-\mathrm{H}), 7.76(2 \mathrm{H}$, $\mathrm{d}, J=8.5 \mathrm{~Hz}, \mathrm{Ar}-\mathrm{H}), 7.95-7.99(5 \mathrm{H}, \mathrm{m}, \mathrm{Ar}-\mathrm{H}), 8.11(1 \mathrm{H}$, $\mathrm{d}, J=5 \mathrm{~Hz}, \mathrm{Ar}-\mathrm{H}), 9.90(\mathrm{H}, \mathrm{s}, \mathrm{N}-\mathrm{H})$. For $\mathrm{C}_{27} \mathrm{H}_{27} \mathrm{~N}_{5} \mathrm{O}_{2} \mathrm{~S}$ 
calculated: (\%) C 66.78, H 5.60, N 14.42; found: (\%) C 66.85, H 5.68, N 14.50. MS: $m / z 486(\mathrm{M}+1)$.

2.2.7.2-[4-(Pyrimidin-2-yl)piperazin-1-yl]-N-[4-(2-(4-methoxyphenyl)thiazol-4-yl)phenyl]acetamide (6g). Yield 78-80\%, m.p. $160-163^{\circ} \mathrm{C}$. IR (KBr) $v_{\max }\left(\mathrm{cm}^{-1}\right): 3315$ (amide $\mathrm{N}-\mathrm{H}$ ), 3038 (aromatic C-H), 2951 (aliphatic C-H), 1676 (amide $\mathrm{C}=\mathrm{O}), 1596-1307 \quad(\mathrm{C}=\mathrm{C}$ and $\mathrm{C}=\mathrm{N}), 1261-978 \quad(\mathrm{C}-\mathrm{O}$ and C-N). ${ }^{1} \mathrm{H}-\mathrm{NMR}\left(500 \mathrm{MHz}, \mathrm{DMSO}-d_{6}, \mathrm{ppm}\right): \delta 2.59(4 \mathrm{H}, \mathrm{t}$, $\left.J=5 \mathrm{~Hz}, \mathrm{~N}-\mathrm{CH}_{2}\right), 3.21\left(2 \mathrm{H}, \mathrm{s}, \mathrm{COCH}_{2}\right), 3.81(4 \mathrm{H}, \mathrm{t}, J=5 \mathrm{~Hz}$, $\left.\mathrm{N}-\mathrm{CH}_{2}\right), 3.83\left(3 \mathrm{H}, \mathrm{s}, \mathrm{OCH}_{3}\right), 6.63(1 \mathrm{H}, \mathrm{t}, J=4.5 \mathrm{~Hz}, \mathrm{Ar}-\mathrm{H})$, $7.08(2 \mathrm{H}, \mathrm{d}, J=9 \mathrm{~Hz}, \mathrm{Ar}-\mathrm{H}), 7.76(2 \mathrm{H}, \mathrm{d}, J=9 \mathrm{~Hz}, \mathrm{Ar}-\mathrm{H})$, 7.95-7.96 (5H, m, Ar-H), $8.36(2 \mathrm{H}, \mathrm{d}, J=5 \mathrm{~Hz}, \mathrm{Ar}-\mathrm{H}), 9.91$ (1H, s, N-H). For $\mathrm{C}_{26} \mathrm{H}_{26} \mathrm{~N}_{6} \mathrm{O}_{2} \mathrm{~S}$ calculated: (\%) C 64.18, $\mathrm{H}$ 5.39, N 17.27; found: (\%) C 64.25, H 5.44, N 17.32. MS: $m / z$ $487(\mathrm{M}+1)$.

2.2.8. 2-[4-(2-Furoyl)piperazin-1-yl]-N-[4-(2-(4-methoxyphenyl)thiazol-4-yl)phenyl]acetamide (6h). Yield 74-76\%, m.p. $136-138^{\circ} \mathrm{C}$. IR (KBr) $v_{\max }\left(\mathrm{cm}^{-1}\right): 3279$ (amide $\left.\mathrm{N}-\mathrm{H}\right), 3063$ (aromatic C-H), 2927 (aliphatic C-H), 1658 (amide $\mathrm{C}=\mathrm{O}$ ), 1604-1306 $(\mathrm{C}=\mathrm{C}$ and $\mathrm{C}=\mathrm{N}), 1236-978(\mathrm{C}-\mathrm{O}$ and $\mathrm{C}-\mathrm{N}) .{ }^{1} \mathrm{H}-$ NMR $\left(500 \mathrm{MHz}, \mathrm{DMSO}-d_{6}, \mathrm{ppm}\right): \delta 2.61(4 \mathrm{H}, \mathrm{t}, J=5 \mathrm{~Hz}$, $\left.\mathrm{N}-\mathrm{CH}_{2}\right), 3.24\left(2 \mathrm{H}, \mathrm{s}, \mathrm{COCH}_{2}\right), 3.76\left(4 \mathrm{H}, \mathrm{t}, J=5 \mathrm{~Hz}, \mathrm{~N}-\mathrm{CH}_{2}\right)$, $3.87\left(3 \mathrm{H}, \mathrm{s}, \mathrm{OCH}_{3}\right), 6.63-6.64(1 \mathrm{H}, \mathrm{m}, \mathrm{Ar}-\mathrm{H}), 7.01(1 \mathrm{H}, \mathrm{d}$, $J=3.5 \mathrm{~Hz}, \mathrm{Ar}-\mathrm{H}), 7.08(2 \mathrm{H}, \mathrm{d}, J=9 \mathrm{~Hz}, \mathrm{Ar}-\mathrm{H}), 7.77(2 \mathrm{H}$, d, $J=8.5 \mathrm{~Hz}, \mathrm{Ar}-\mathrm{H}), 7.85(1 \mathrm{H}, \mathrm{s}, \mathrm{Ar}-\mathrm{H}), 7.96-8.01(5 \mathrm{H}, \mathrm{m}$, Ar-H), $9.94(1 \mathrm{H}, \mathrm{s}, \mathrm{N}-\mathrm{H}) .{ }^{13} \mathrm{C}-\mathrm{NMR}\left(125 \mathrm{MHz}\right.$, DMSO- $d_{6}$, ppm): $\delta 53.18,53.55,55.87,61.82,111.76,112.85,115.06,115.40$, $115.99,120.08,126.37,126.96,128.25,129.84,139.00,145.15$, 147.48 , 155.17, 158.79, 161.41, 167.21, 168.66. For $\mathrm{C}_{27} \mathrm{H}_{26} \mathrm{~N}_{4} \mathrm{O}_{4} \mathrm{~S}$ calculated: (\%) C 64.52, H 5.21, N 11.15; found: (\%) C 64.46, H 5.29, N 11.24. MS: $m / z 503(M+1)$.

2.2.9. 2-(4-Benzylpiperazin-1-yl)-N-[4-(2-(4-chlorophenyl)thiazol-4-yl)phenyl]acetamide (6i). Yield 70-73\%, m.p. 171$173^{\circ} \mathrm{C}$. IR $(\mathrm{KBr}) v_{\max }\left(\mathrm{cm}^{-1}\right)$ : 3288 (amide $\left.\mathrm{N}-\mathrm{H}\right), 3013$ (aromatic C-H), 2966 (aliphatic C-H), 1664 (amide C=O), 1591$1315(\mathrm{C}=\mathrm{C}$ and $\mathrm{C}=\mathrm{N}), 1234-978(\mathrm{C}-\mathrm{O}$ and $\mathrm{C}-\mathrm{N}) .{ }^{1} \mathrm{H}-\mathrm{NMR}$ $\left(500 \mathrm{MHz}, \mathrm{DMSO}-d_{6}, \mathrm{ppm}\right): \delta 2.50\left(4 \mathrm{H}, \mathrm{brs}, \mathrm{N}-\mathrm{CH}_{2}\right), 3.14$ $\left(2 \mathrm{H}, \mathrm{s}, \mathrm{COCH}_{2}\right), 3.48\left(2 \mathrm{H}, \mathrm{s}, \mathrm{C}-\mathrm{CH}_{2}\right), 7.08(2 \mathrm{H}, \mathrm{d}, J=9 \mathrm{~Hz}$, Ar-H), 7.29-7.32 (5H, m, Ar-H), $7.60(2 \mathrm{H}, \mathrm{d}, J=8.5 \mathrm{~Hz}$, $\mathrm{Ar}-\mathrm{H}), 7.75(2 \mathrm{H}, \mathrm{d}, J=8.5 \mathrm{~Hz}, \mathrm{Ar}-\mathrm{H}), 7.97(1 \mathrm{H}, \mathrm{d}, J=8.5 \mathrm{~Hz}$, Ar-H), $8.04(1 \mathrm{H}, \mathrm{d}, J=8.5 \mathrm{~Hz}, \mathrm{Ar}-\mathrm{H}), 8.11(1 \mathrm{H}, \mathrm{s}, \mathrm{Ar}-\mathrm{H}), 9.82$ $(1 \mathrm{H}, \mathrm{s}, \mathrm{N}-\mathrm{H})$. For $\mathrm{C}_{28} \mathrm{H}_{27} \mathrm{ClN}_{4} \mathrm{OS}$ calculated: (\%) C 66.85, $\mathrm{H}$ 5.41, N 11.14; found: (\%) C 66.94, H 5.47, N 11.20. MS: $m / z$ $502.5(\mathrm{M}+1)$.

2.2.10. 2-[4-(Pyridin-2-yl)piperazin-1-yl]-N-[4-(2-(4-chlorophenyl)thiazol-4-yl)phenyl]acetamide (6j). Yield 73-76\%, m.p. $220-225^{\circ} \mathrm{C}$. IR $(\mathrm{KBr}) v_{\max }\left(\mathrm{cm}^{-1}\right): 3300($ amide N-H), 3002 (aromatic C-H), 2924 (aliphatic C-H), 1666 (amide $\mathrm{C}=\mathrm{O}), 1591-1305 \quad(\mathrm{C}=\mathrm{C}$ and $\mathrm{C}=\mathrm{N}), 1244-1018 \quad(\mathrm{C}-\mathrm{O}$ and C-N). ${ }^{1} \mathrm{H}-\mathrm{NMR}\left(500 \mathrm{MHz}, \mathrm{DMSO}-d_{6}, \mathrm{ppm}\right): \delta 2.63(4 \mathrm{H}$, $\left.\mathrm{t}, J=4.5 \mathrm{~Hz}, \mathrm{~N}-\mathrm{CH}_{2}\right), 3.22\left(2 \mathrm{H}, \mathrm{s}, \mathrm{COCH}_{2}\right), 3.56(4 \mathrm{H}, \mathrm{t}$, $\left.J=4.5 \mathrm{~Hz}, \mathrm{~N}-\mathrm{CH}_{2}\right), 6.63-6.65(1 \mathrm{H}, \mathrm{m}, \mathrm{Ar}-\mathrm{H}), 6.83(1 \mathrm{H}, \mathrm{d}$, $J=8.5 \mathrm{~Hz}, \mathrm{Ar}-\mathrm{H}), 7.53(1 \mathrm{H}, \mathrm{t}, J=4.5 \mathrm{~Hz}, \mathrm{Ar}-\mathrm{H}), 7.60(2 \mathrm{H}, \mathrm{d}$, $J=8.5 \mathrm{~Hz}, \mathrm{Ar}-\mathrm{H}), 7.78(2 \mathrm{H}, \mathrm{d}, J=8.5 \mathrm{~Hz}, \mathrm{Ar}-\mathrm{H}), 8.03(2 \mathrm{H}, \mathrm{d}$,
$J=8.5 \mathrm{~Hz}, \mathrm{Ar}-\mathrm{H}), 8.06(2 \mathrm{H}, \mathrm{d}, J=8.5 \mathrm{~Hz}, \mathrm{Ar}-\mathrm{H}), 8.12(2 \mathrm{H}$, s, Ar-H), $9.92(1 \mathrm{H}, \mathrm{s}, \mathrm{N}-\mathrm{H}) .{ }^{13} \mathrm{C}-\mathrm{NMR}\left(125 \mathrm{MHz}, \mathrm{DMSO}-d_{6}\right.$, ppm): $\delta 45.05,52.98,62.25,107.58,113.45,114.44,120.06$, $127.05,128.37,129.53,129.81,132.34,135.33,137.98,139.19$, $148.04,155.60,159.51,165.95,168.80$. For $\mathrm{C}_{26} \mathrm{H}_{24} \mathrm{ClN}_{5} \mathrm{OS}$ calculated: (\%) C 63.73, H 4.94, N 14.29; found: (\%) C 63.81, H 4.98, N 14.32. MS: $m / z 489.5(M+1)$.

2.2.11. 2-[4-(Pyrimidin-2-yl)piperazin-1-yl]-N-[4-(2-(4-chlorophenyl)thiazol-4-yl)phenyl]acetamide (6k). Yield $70-74 \%$, m.p. $196-198^{\circ} \mathrm{C}$. IR (KBr) $v_{\max }\left(\mathrm{cm}^{-1}\right): 3290$ (amide N-H), 3022 (aromatic C-H), 2931 (aliphatic C-H), 1670 (amide $\mathrm{C}=\mathrm{O}), 1585-1309(\mathrm{C}=\mathrm{C}$ and $\mathrm{C}=\mathrm{N}), 1256-979 \quad(\mathrm{C}-\mathrm{O}$ and C-N). ${ }^{1} \mathrm{H}-\mathrm{NMR}\left(500 \mathrm{MHz}, \mathrm{DMSO}-d_{6}, \mathrm{ppm}\right): \delta 2.59(4 \mathrm{H}$, $\left.\mathrm{t}, J=4.5 \mathrm{~Hz}, \mathrm{~N}-\mathrm{CH}_{2}\right), 3.21\left(2 \mathrm{H}, \mathrm{s}, \mathrm{COCH}_{2}\right), 3.81(4 \mathrm{H}, \mathrm{t}$, $\left.J=4.5 \mathrm{~Hz}, \mathrm{~N}-\mathrm{CH}_{2}\right), 6.62(1 \mathrm{H}, \mathrm{t}, J=5 \mathrm{~Hz}, \mathrm{Ar}-\mathrm{H}), 7.59(2 \mathrm{H}, \mathrm{d}$, $J=8.5 \mathrm{~Hz}, \mathrm{Ar}-\mathrm{H}), 7.77(2 \mathrm{H}, \mathrm{d}, J=8.5 \mathrm{~Hz}, \mathrm{Ar}-\mathrm{H}), 8.02(2 \mathrm{H}$, d, $J=8.5 \mathrm{~Hz}, \operatorname{Ar}-\mathrm{H}), 8.05(2 \mathrm{H}, \mathrm{d}, J=8.5 \mathrm{~Hz}, \mathrm{Ar}-\mathrm{H}), 8.12(1 \mathrm{H}$, s, Ar-H), $8.35(2 \mathrm{H}, \mathrm{d}, J=4.5 \mathrm{~Hz}, \mathrm{Ar}-\mathrm{H}), 9.92(1 \mathrm{H}, \mathrm{s}, \mathrm{N}-\mathrm{H})$. For $\mathrm{C}_{25} \mathrm{H}_{23} \mathrm{ClN}_{6} \mathrm{OS}$ calculated: (\%) C 61.15, H 4.72, N 17.12; found: (\%) C 61.23, H 4.80, N 17.16. MS: $m / z$ 490.5 (M + 1).

2.2.12.2-[4-(2-Furoyl)piperazin-1-yl]-N-[4-(2-(4-chlorophenyl)thiazol-4-yl)phenyl]acetamide (6l). Yield $72-75 \%$, m.p. 139-141 ${ }^{\circ} \mathrm{C}$. IR (KBr) $v_{\max }\left(\mathrm{cm}^{-1}\right): 3317$ (amide $\left.\mathrm{N}-\mathrm{H}\right), 3042$ (aromatic C-H), 2962 (aliphatic C-H), 1676 (amide $\mathrm{C}=\mathrm{O}$ ), 1625-1305 $(\mathrm{C}=\mathrm{C}$ and $\mathrm{C}=\mathrm{N}), 1261-978(\mathrm{C}-\mathrm{O}$ and $\mathrm{C}-\mathrm{N}) .{ }^{1} \mathrm{H}-$ NMR (500 MHz, DMSO- $\left.d_{6}, \mathrm{ppm}\right): \delta 2.60(4 \mathrm{H}, \mathrm{t}, J=5 \mathrm{~Hz}$, $\left.\mathrm{N}-\mathrm{CH}_{2}\right), 3.34\left(2 \mathrm{H}, \mathrm{s}, \mathrm{COCH}_{2}\right), 3.74\left(4 \mathrm{H}, \mathrm{t}, J=5 \mathrm{~Hz}, \mathrm{~N}-\mathrm{CH}_{2}\right)$, 6.62-6.63 (1H, m, Ar-H), $7.01(1 \mathrm{H}, \mathrm{d}, J=3.5 \mathrm{~Hz}, \mathrm{Ar}-\mathrm{H}), 7.59$ $(2 \mathrm{H}, \mathrm{d}, J=8.5 \mathrm{~Hz}, \mathrm{Ar}-\mathrm{H}), 7.76-7.83(2 \mathrm{H}, \mathrm{m}, \mathrm{Ar}-\mathrm{H}), 7.95-8.15$ $(6 \mathrm{H}, \mathrm{s}, \operatorname{Ar}-\mathrm{H}), 9.96(1 \mathrm{H}, \mathrm{s}, \mathrm{N}-\mathrm{H})$. For $\mathrm{C}_{26} \mathrm{H}_{23} \mathrm{ClN}_{4} \mathrm{O}_{3} \mathrm{~S}$ calculated: (\%) C 61.59, H 4.57, N 11.05; found: (\%) C 61.65, H 4.63, N 11.12. MS: $m / z 506.5(\mathrm{M}+1)$.

2.2.13. 2-(4-Benzylpiperazin-1-yl)-N-[4-(2-(4-florophenyl)thiazol-4-yl)phenyl]acetamide (6m). Yield 69-73\%, m.p. 171$172^{\circ} \mathrm{C}$. IR (KBr) $v_{\max }\left(\mathrm{cm}^{-1}\right): 3317$ (amide $\left.\mathrm{N}-\mathrm{H}\right), 3015$ (aromatic C-H), 2970 (aliphatic C-H), 1678 (amide $\mathrm{C}=\mathrm{O}$ ), 1502-1306 $(\mathrm{C}=\mathrm{C}$ and $\mathrm{C}=\mathrm{N}), 1234-978(\mathrm{C}-\mathrm{O}$ and $\mathrm{C}-\mathrm{N}) .{ }^{1} \mathrm{H}-$ NMR $\left(500 \mathrm{MHz}, \mathrm{DMSO}-d_{6}, \mathrm{ppm}\right): \delta 2.45(4 \mathrm{H}, \mathrm{t}, J=5 \mathrm{~Hz}$, $\left.\mathrm{N}-\mathrm{CH}_{2}\right), 2.54\left(4 \mathrm{H}, \mathrm{t}, J=4.5 \mathrm{~Hz}, \mathrm{~N}-\mathrm{CH}_{2}\right), 3.14(2 \mathrm{H}, \mathrm{s}$, $\left.\mathrm{COCH}_{2}\right), 3.48\left(2 \mathrm{H}, \mathrm{s}, \mathrm{C}-\mathrm{CH}_{2}\right), 7.24-7.39(7 \mathrm{H}, \mathrm{m}, \mathrm{Ar}-\mathrm{H})$, $7.73(2 \mathrm{H}, \mathrm{d}, J=8.5 \mathrm{~Hz}, \mathrm{Ar}-\mathrm{H}), 7.99(2 \mathrm{H}, \mathrm{d}, J=8.5 \mathrm{~Hz}$, Ar-H), 8.06-8.11 (3H, m, Ar-H), $9.85(1 \mathrm{H}, \mathrm{s}, \mathrm{N}-\mathrm{H})$. For $\mathrm{C}_{28} \mathrm{H}_{27} \mathrm{FN}_{4}$ OS calculated: (\%) C 69.11, H 5.59, N 11.51; found: (\%) C 69.18, H 5.65, N 11.63. MS: $m / z 487(\mathrm{M}+1)$.

2.2.14. 2-[4-(Pyridin-2-yl)piperazin-1-yl]-N-[4-(2-(4-florophenyl)thiazol-4-yl)phenyl]acetamide (6n). Yield 70-74\%, m.p. 163-164 ${ }^{\circ} \mathrm{C}$. IR $(\mathrm{KBr}) v_{\max }\left(\mathrm{cm}^{-1}\right): 3317$ (amide N-H), 3023 (aromatic C-H), 2987 (aliphatic C-H), 1678 (amide C=O), 1591-1305 (C=C and $\mathrm{C}=\mathrm{N}), 1247-978(\mathrm{C}-\mathrm{O}$ and $\mathrm{C}-\mathrm{N}) .{ }^{1} \mathrm{H}-$ $\operatorname{NMR}\left(500 \mathrm{MHz}, \mathrm{DMSO}-d_{6}, \mathrm{ppm}\right): \delta 2.63(4 \mathrm{H}, \mathrm{t}, J=4.5 \mathrm{~Hz}$, $\left.\mathrm{N}-\mathrm{CH}_{2}\right), 3.22\left(2 \mathrm{H}, \mathrm{s}, \mathrm{COCH}_{2}\right), 3.56\left(4 \mathrm{H}, \mathrm{t}, J=5 \mathrm{~Hz}, \mathrm{~N}-\mathrm{CH}_{2}\right)$, 6.62-6.65 $(1 \mathrm{H}, \mathrm{m}, \mathrm{Ar}-\mathrm{H}), 6.83(1 \mathrm{H}, \mathrm{d}, J=8.5 \mathrm{~Hz}, \mathrm{Ar}-\mathrm{H})$, $7.35-7.39(2 \mathrm{H}, \mathrm{m}, \mathrm{Ar}-\mathrm{H}), 7.53(1 \mathrm{H}, \mathrm{t}, J=4.5 \mathrm{~Hz}, \mathrm{Ar}-\mathrm{H})$, $7.76(2 \mathrm{H}, \mathrm{d}, J=8.5 \mathrm{~Hz}, \mathrm{Ar}-\mathrm{H}), 7.99(2 \mathrm{H}, \mathrm{d}, J=8.5 \mathrm{~Hz}$, 
Ar-H), 8.06-8.12 (4H, m, Ar-H), 9.91 (1H, s, N-H). For $\mathrm{C}_{26} \mathrm{H}_{24} \mathrm{FN}_{5} \mathrm{OS}$ calculated: (\%) C 65.94, H 5.11, N 14.79; found: (\%) C 65.86, H 5.18, N 14.65. MS: $m / z 474(\mathrm{M}+1)$.

2.2.15. 2-[4-(Pyrimidin-2-yl)piperazin-1-yl]-N-[4-(2-(4-florophenyl)thiazol-4-yl)phenyl]acetamide (6o). Yield 70-74\%, m.p. $187-188^{\circ} \mathrm{C}$. IR $(\mathrm{KBr}) v_{\max }\left(\mathrm{cm}^{-1}\right): 3317$ (amide $\left.\mathrm{N}-\mathrm{H}\right)$, 3036 (aromatic C-H), 2954 (aliphatic C-H), 1677 (amide $\mathrm{C}=\mathrm{O}), 1583-1307 \quad(\mathrm{C}=\mathrm{C}$ and $\mathrm{C}=\mathrm{N}), 1257-978 \quad(\mathrm{C}-\mathrm{O}$ and C-N). ${ }^{1} \mathrm{H}-\mathrm{NMR}\left(500 \mathrm{MHz}, \mathrm{DMSO}-d_{6}, \mathrm{ppm}\right): \delta 2.61(4 \mathrm{H}$, $\left.\mathrm{t}, J=3 \mathrm{~Hz}, \mathrm{~N}-\mathrm{CH}_{2}\right), 3.22\left(2 \mathrm{H}, \mathrm{s}, \mathrm{COCH}_{2}\right), 3.83(4 \mathrm{H}, \mathrm{t}$, $\left.J=4.5 \mathrm{~Hz}, \mathrm{~N}-\mathrm{CH}_{2}\right), 6.63(1 \mathrm{H}, \mathrm{t}, J=4.5 \mathrm{~Hz}, \mathrm{Ar}-\mathrm{H}), 7.50-7.56$ $(3 \mathrm{H}, \mathrm{m}, \mathrm{Ar}-\mathrm{H}), 7.78(2 \mathrm{H}, \mathrm{d}, J=9 \mathrm{~Hz}, \mathrm{Ar}-\mathrm{H}), 7.98-8.06(4 \mathrm{H}$, m, Ar-H), 8.09 (1H, s, Ar-H), 8.37 (2H, d, $J=8.5 \mathrm{~Hz}, \mathrm{Ar}-\mathrm{H})$, $9.93(1 \mathrm{H}, \mathrm{s}, \mathrm{N}-\mathrm{H}) .{ }^{13} \mathrm{C}-\mathrm{NMR}\left(125 \mathrm{MHz}, \mathrm{DMSO}-d_{6}, \mathrm{ppm}\right): \delta$ 43.71, 52.97, 62.19, 110.59, 114.03, 116.68, 116.85, 120.07, 127.02, $128.95,129.02,129.62,130.19,139.14,155.44,158.40,161.70$, 162.74, 166.13, 168.80. For $\mathrm{C}_{25} \mathrm{H}_{23} \mathrm{FN}_{6} \mathrm{OS}$ calculated: (\%) C 63.27, H 4.89, N 17.71; found: (\%) C 63.19, H 4.94, N 17.66. MS: $m / z 475(\mathrm{M}+1)$.

2.2.16. 2-[4-(2-Furoyl)piperazin-1-yl]-N-[4-(2-(4-florophen$y$ l)thiazol-4-yl)phenyl]acetamide (6p). Yield 74-78\%, m.p. $146-148^{\circ} \mathrm{C}$. IR (KBr) $v_{\max }\left(\mathrm{cm}^{-1}\right): 3319$ (amide N-H), 3031 (aromatic C-H), 2927 (aliphatic C-H), 1674 (amide $\mathrm{C}=\mathrm{O}$ ), 1589-1307 ( $\mathrm{C}=\mathrm{C}$ and $\mathrm{C}=\mathrm{N}), 1232-978(\mathrm{C}-\mathrm{O}$ and $\mathrm{C}-\mathrm{N}) .{ }^{1} \mathrm{H}-$ NMR (500 MHz, DMSO- $\left.d_{6}, \mathrm{ppm}\right): \delta 2.60(4 \mathrm{H}, \mathrm{t}, J=5 \mathrm{~Hz}$, $\left.\mathrm{N}-\mathrm{CH}_{2}\right), 3.34\left(2 \mathrm{H}, \mathrm{s}, \mathrm{COCH}_{2}\right), 3.74(4 \mathrm{H}, \mathrm{t}, J=5 \mathrm{~Hz}, \mathrm{~N}-$ $\left.\mathrm{CH}_{2}\right), 6.61-6.62(1 \mathrm{H}, \mathrm{m}, \mathrm{Ar}-\mathrm{H}), 7.03(1 \mathrm{H}, \mathrm{d}, J=3.5 \mathrm{~Hz}, \mathrm{Ar}-$ $\mathrm{H}), 7.61(2 \mathrm{H}, \mathrm{d}, J=8.5 \mathrm{~Hz}, \mathrm{Ar}-\mathrm{H}), 7.75-7.80(2 \mathrm{H}, \mathrm{m}, \mathrm{Ar}-\mathrm{H})$, 7.98-8.16 (6H, s, Ar-H), $9.92(1 \mathrm{H}, \mathrm{s}, \mathrm{N}-\mathrm{H})$. For $\mathrm{C}_{26} \mathrm{H}_{23} \mathrm{FN}_{4} \mathrm{O}_{3} \mathrm{~S}$ calculated: (\%) C 63.66, H 4.73, N 11.42; found: (\%) C 63.72, $\mathrm{H}$ 4.81, N 11.46. MS: $m / z 491(\mathrm{M}+1)$.

2.3. Antimicrobial Activity. The study was designed to compare MICs obtained by the CLSI reference M7-A7 broth microdilution method [15]. MIC readings were performed twice for each chemical agent. Final products were tested for their in vitro growth inhibitory activity against human pathogenic microorganisms as Gram-positive bacteria, Enterococcus faecalis (ATCC 29212) and as Gram-negative bacteria, Pseudomonas aeruginosa (ATCC 27853), Klebsiella pneumoniae (ATCC 700603), Escherichia coli (ATCC 35218), and E. coli (ATCC 25922) and yeast as Candida albicans (90028), Candida glabrata (ATCC 90030), Candida krusei (ATCC 6258), and Candida parapsilosis (ATCC 7330). Chloramphenicol and ketoconazole were used as control drugs.

2.4. Anticholinesterase Activity. The inhibition of acetylcholinesterase enzyme, AChE (E.C.3.1.1.7 from Electric Eel, 500 units), was determined spectrophotometrically using acetylthiocholine (ATC) as substrate according to the modified method of Ellman's test [16]. Enzyme solutions were prepared in gelatin solution (1\%), at a concentration of 2.5 units $/ \mathrm{mL}$. AChE and compound solution $(50 \mu \mathrm{L})$ which is prepared in $2 \%$ dimethyl sulfoxide at 0.1 and $1 \mathrm{mM}$ concentrations were added to $3.0 \mathrm{~mL}$ phosphate buffer $(\mathrm{pH}$ $8 \pm 0.1)$ and incubated at $25^{\circ} \mathrm{C}$ for $5 \mathrm{~min}$. The reaction was started by adding 5,5-dithio-bis(2-nitrobenzoic)acid, DTNB $(50 \mu \mathrm{L})$, and ATC $(10 \mu \mathrm{L})$ to the enzyme-inhibitor mixture. Spectrophotometric measurements were performed on a 1700 Shimadzu UV-1700 UV-Vis spectrophotometer at $412 \mathrm{~nm}$. Donepezil hydrochloride was used as a positive control and all processes were assayed in triplicate [17].

\section{Results and Discussion}

The final compounds (6a-6n) were synthesized by a five-step (Figure 1), convenient synthetic routeaccording to the literaturereported before [14]. Different 4-substituted thiobenzamides $\left(\mathrm{H}, \mathrm{OCH}_{3}, \mathrm{Cl}, \mathrm{F}\right)$ were used as starting materials. They have reacted with $\mathrm{N}$-[4-(2-bromoacetyl)phenyl] acetamide to obtain thiazole compounds (3). After deacetylation of these intermediates, 2-chloro- $N$-[4-(2-(4-substituted phenyl)-4thiazolyl)phenyl]acetamides (5) were gained with chloroacetyl chloride. Finally, 2-[4-(aryl)piperazin-1-yl]-N-[4-(2(4-substituted phenyl)thiazol-4-yl)phenyl]acetamide derivatives $(6 \mathbf{6}-\mathbf{6 n})$ were afforded by the reaction of compound 5 and corresponding piperazine derivatives. Synthetic protocol for the compounds is outlined in Figure 1. Structure elucidations of the final compounds were performed with IR, ${ }^{1} \mathrm{H}$ NMR, and MS spectroscopic methods. In the IR spectra of the final compounds, characteristic stretching bands were observed at $3253-3319 \mathrm{~cm}^{-1}$ for N-H bond, $1303-1625 \mathrm{~cm}^{-1}$ for $\mathrm{C}=\mathrm{C}$ and $\mathrm{C}=\mathrm{N}$ bonds, $978-1263 \mathrm{~cm}^{-1}$ for $\mathrm{C}-\mathrm{N}$ and $\mathrm{C}-$ $\mathrm{O}$ bonds, and $1653-1678 \mathrm{~cm}^{-1}$ for $\mathrm{C}=\mathrm{O}$ bond, respectively. In the NMR spectra of the compounds $\mathbf{6 a - 6 n}$, protons of piperazine $\mathrm{CH}_{2}$ were observed at about $2.50-2.63 \mathrm{ppm}$ and 3.56-3.82 ppm ranges as triplets or broad singlets. The protons bonded to carbonyl were assigned at 3.14-3.34 ppm and heterocyclic rings and chloro/fluoro substituted phenyl rings were given peaks near to downfield region. Aromatic protons resonated at a large area between 6.62 and $8.37 \mathrm{ppm}$ and amide protons were determined at about 9.82-9.96 ppm, as singlet peaks. Regarding to the mass spectra, all final compounds exhibited similar behavior in their fragmentation pattern, showing the molecular ion peak.

All final compounds were screened for their acetylcholinesterase inhibitory activities and antimicrobial activities. Initially, 2-(4-arylpiperazine-1-yl)- $N$-[4-(2-(4-substituted phenyl)thiazol-4-yl)phenyl]acetamide derivatives (6a-6p) have been designed and synthesizedfor investigation of their potential anticholinesterase activity. According to our previous study [14], 2-(4-substituted piperazine-1-yl)- $N$-[4-(2methylthiazol-4-yl)phenyl]acetamide derivatives based on the structure of AChE inhibitors donepezil, BYYT-25 and acotiamide were investigated and compounds with pyridyl, benzyl, and furoyl piperazine substituents were observed with high anticholinesterase activity. This study was planned to improve new anticholinesterase compounds using the same skeleton, but with different substitutions. However, in contrast to expectations the synthesized compounds (6a-6n) did not show any noticeable anticholinesterase activity (Table 2). It is evident that phenyl/4-substituted phenyl moiety at the second position of thiazole ring has eliminated activity. Only 

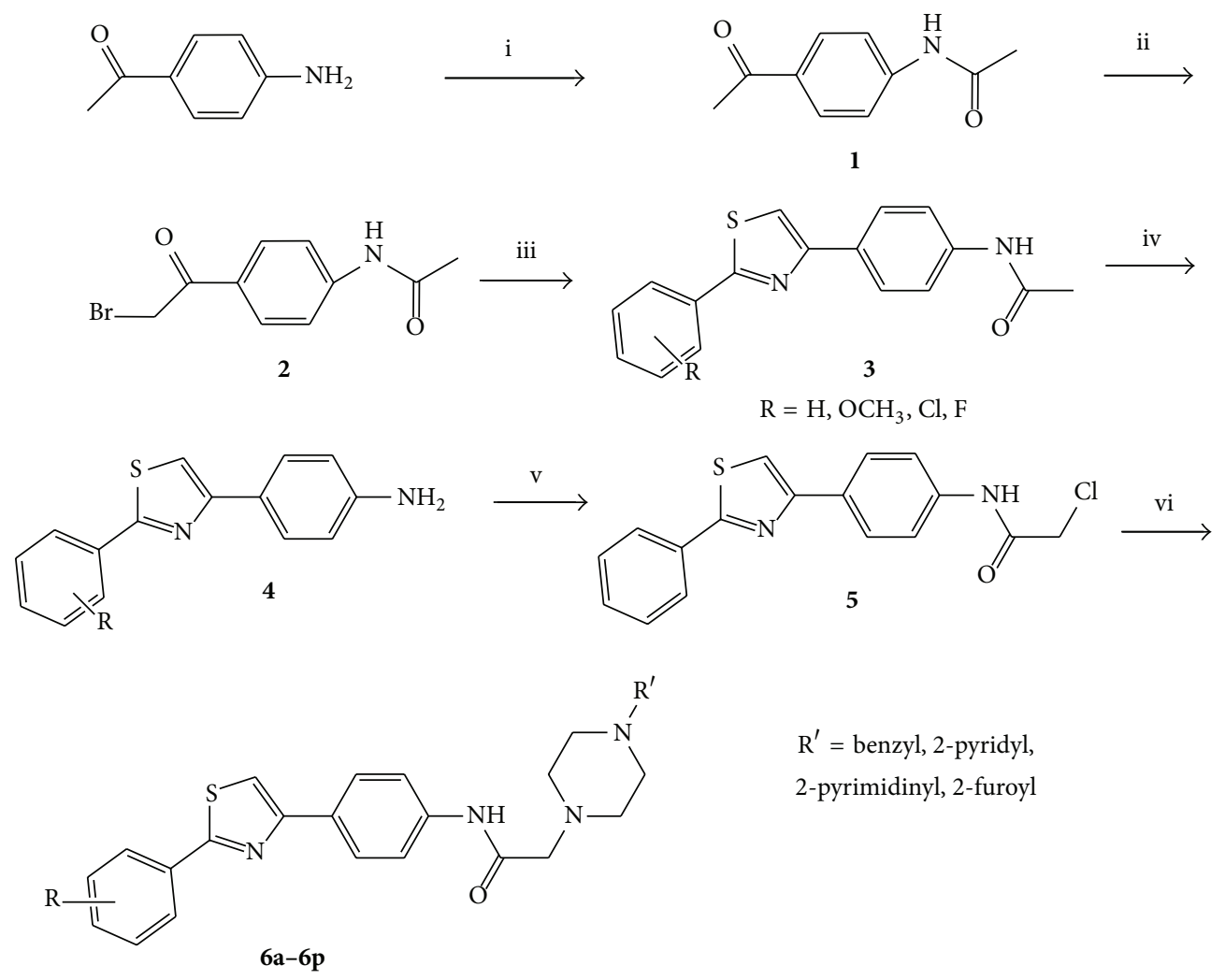

FIgure 1: Synthesis of the compounds(6a-6p). Reagents are (i) acetyl chloride, TEA, THF, 0-5 $5^{\circ}$; (ii) $\mathrm{Br}_{2}$, $\mathrm{AcOH}$; (iii) thiobenzamide derivatives, EtOH, r.t.; (iv) $10 \% \mathrm{HCl}$, EtOH, reflux; (v) chloroacetyl chloride, TEA, THF, r.t.; and (vi) appropriate 4-substituted piperazine derivatives, $\mathrm{K}_{2} \mathrm{CO}_{3}$, acetone, reflux.

two compounds $\mathbf{6 j}$ and $\mathbf{6 m}$ have exhibited inhibition over $50 \%$ at the highest tested dose $(1 \mathrm{mM})$.

Whereupon considering many studies on thiazole derivatives biological profile, we tend to screen antimicrobial activity of the synthesized 2,4-disubstituted thiazole compounds. Sixteen new compounds were tested for their antibacterial and antifungal activities. The antifungal activity of the compounds was found to be higher than their antimicrobial activities (Table 1). Compounds $\mathbf{6 c}$ and $\mathbf{6 d}$ showed higher activity than the other compounds against E. faecalis which is a Gram-positive bacterium. Among the tested Gram-negative bacteria, compounds $\mathbf{6 c}-\mathbf{6 f}, \mathbf{6 i}$ and $\mathbf{6 j}$ exhibited the highest activity against $K$. pneumonia with a MIC value of $100 \mu \mathrm{g} / \mathrm{mL}$ which is two-fold standard drug's MIC value. Compound $\mathbf{6 1}$ showed equipotent activity to standard drug againstanother Gram-negative bacterium $P$. aeruginosa. Against two tested E. coli species (ATCC 35218 and ATCC 25922), only compounds $\mathbf{6 d}$ and $\mathbf{6 m}$ displayed moderate antibacterial activity compared with standard drug chloramphenicol. As for the antifungal activity, compounds showed higher activities against four tested Candida species. Compounds $\mathbf{6 a}, \mathbf{6 b}, \mathbf{6 i}$, and $\mathbf{6 m}$ exhibited same efficiency with ketoconazole against $C$. albicans and also compound 6f towards C. glabrata. Against C. krusei, the MIC value was found as $50 \mu \mathrm{g} / \mathrm{mL}$ for compound $\mathbf{6 a}$ which was very high according to ketoconazole's MIC value $(3.13 \mu \mathrm{g} / \mathrm{mL})$.
Among all Candida species, the most sensitive Candida was established as C. parapsilosis. Compounds $\mathbf{6 b}, \mathbf{6} \mathbf{c}$, and $\mathbf{6 h}$ were found twice as active as standard drug, whereas compounds 6a, 6d, 6e, 6f, 6m, and $\mathbf{6 n}$ showed equipotent activity. All the other compounds displayed half the potency of ketoconazole against the same microorganism.

The structures of the tested compounds were based on a thiazole ring which has phenyl moiety at second and fourth positions of it. The substitutions of the phenyl ring at second position are methoxy, chloro and fluoro groups. The difference at the fourth position of the thiazole ring is pyridyl, pyrimidinyl benzyl, and furoyl piperazine moieties bonded to phenyl ring with an amide linker. 2-Furoyl piperazine substitution has attracted attention for its antibacterial activity. In antifungal activity evaluation, nonsubstituted phenyl group and different substituted piperazine substitutions were observed with significant activity according to Candida species.

\section{Conclusion}

Sixteen new 2-(4-substituted phenyl)-4-(acetamidophenyl)thiazole including compounds were synthesized. All final compounds were screened for their antibacterial, antifungal, and anticholinesterase activities. None of the compounds showed prominent anticholinesterase activity. Antifungal 
TABLE 1: Antimicrobial activity of the compounds 6a-6p (MIC, $\mu \mathrm{g} / \mathrm{mL})$.

\begin{tabular}{lccccccccc}
\hline Comp. & $\mathrm{A}$ & $\mathrm{B}$ & $\mathrm{C}$ & $\mathrm{D}$ & $\mathrm{E}$ & $\mathrm{F}$ & $\mathrm{G}$ & $\mathrm{H}$ & $\mathrm{I}$ \\
\hline $\mathbf{6 a}$ & 200 & 200 & 200 & 200 & 200 & 50 & 100 & 50 & 100 \\
$\mathbf{6 b}$ & 200 & 200 & 200 & 200 & 200 & 50 & 100 & 100 & 50 \\
$\mathbf{6 c}$ & 100 & 100 & 200 & 200 & 200 & 200 & 100 & 200 & 50 \\
$\mathbf{6 d}$ & 100 & 100 & 200 & 200 & 100 & 200 & 100 & 100 & 100 \\
$\mathbf{6 e}$ & 200 & 100 & 200 & 200 & 400 & 200 & 100 & 100 & 100 \\
$\mathbf{6 f}$ & 200 & 100 & 100 & 200 & 200 & 100 & 50 & 100 & 100 \\
$\mathbf{6 g}$ & 200 & 100 & 100 & 200 & 200 & 100 & 100 & 100 & 200 \\
$\mathbf{6 h}$ & 200 & 200 & 200 & 400 & 200 & 200 & 100 & 100 & 50 \\
$\mathbf{6 i}$ & 200 & 100 & 200 & 200 & 400 & 50 & 100 & 100 & 200 \\
$\mathbf{6 j}$ & 200 & 100 & 200 & 200 & 400 & 100 & 200 & 200 & 200 \\
$\mathbf{6 k}$ & 200 & 200 & 100 & 200 & 400 & 100 & 200 & 200 & 200 \\
$\mathbf{6 l}$ & 200 & 200 & 50 & 200 & 200 & 100 & 200 & 200 & 200 \\
$\mathbf{6 m}$ & 200 & 200 & 200 & 200 & 100 & 50 & 100 & 100 & 100 \\
$\mathbf{6 n}$ & 200 & 200 & 200 & 200 & 200 & 200 & 200 & 200 & 100 \\
$\mathbf{6 o}$ & 200 & 200 & 200 & 200 & 200 & 100 & 200 & 200 & 200 \\
$\mathbf{6 p}$ & 200 & 200 & 200 & 200 & 200 & 200 & 200 & 800 & 200 \\
Ref. 1 & 25 & 50 & 50 & 25 & 25 & - & - & - & - \\
Ref. 2 & - & - & - & - & - & 50 & 50 & 3.13 & 100 \\
\hline
\end{tabular}

Reference 1: Chloramphenicol, Reference 2: Ketoconazole.

A: E. faecalis (ATCC 29212), B: K. pneumonia (ATCC 700603), C: $P$. aeruginosa (ATCC 27853), D: E. coli (ATCC 35218), E: E. coli (ATCC 25922), F: C. albicans (ATCC 90028), G: C. glabrata (ATCC 90030), H: C. krusei (ATCC 6258), I: C. parapsilosis (ATCC 7330).

TABLE 2: \% AChE inbition of the compounds $\mathbf{6 a - 6} \mathbf{p}$ and $\mathrm{IC}_{50}$ values.

\begin{tabular}{lccc}
\hline \multirow{2}{*}{ Compounds } & \multicolumn{2}{c}{$\%$ AChE Inhibition } & \multirow{2}{*}{$\mathrm{IC}_{50}(\mathrm{mM})$} \\
\hline $\mathbf{6 a}$ & $3.45 \pm 5.10$ & - & \\
$\mathbf{6 b}$ & $7.19 \pm 8.45$ & - & \\
$\mathbf{6 c}$ & - & - & \\
$\mathbf{6 d}$ & - & - & \\
$\mathbf{6 e}$ & $10.16 \pm 10.12$ & - & \\
$\mathbf{6 f}$ & $2.86 \pm 2.15$ & - & \\
$\mathbf{6 g}$ & $2.03 \pm 3.21$ & - & \\
$\mathbf{6 h}$ & $7.28 \pm 4.69$ & - & \\
$\mathbf{6 i}$ & $8.63 \pm 3.25$ & - & \\
$\mathbf{6 j}$ & $57.04 \pm 10.65$ & $17.09 \pm 2.3$ & \\
$\mathbf{6 k}$ & $37.09 \pm 5.16$ & $27.78 \pm 5.12$ & \\
$\mathbf{6 l}$ & $29.59 \pm 11.23$ & $5.39 \pm 2.11$ & \\
$\mathbf{6 m}$ & $58.79 \pm 5.61$ & $49.65 \pm 6.49$ & $0.13 \pm 3.74$ \\
$\mathbf{6 n}$ & $43.96 \pm 5.84$ & $40.58 \pm 5.89$ & \\
$\mathbf{6 o}$ & $41.96 \pm 6.89$ & $40.52 \pm 7.36$ & \\
$\mathbf{6 p}$ & $44.68 \pm 2.68$ & $42.92 \pm 3.55$ & \\
Donepezil & $98.24 \pm 1.87$ & $92.23 \pm 2.62$ & $0.054 \pm 0.002(\mu \mathrm{M})$ \\
\hline
\end{tabular}

ND: Not determined. $p<0.01$ (unpaired Student's $t$ test between test compound and Donepezil).

activity of the compounds was found promising. Compounds $\mathbf{6 b}, \mathbf{6 c}$, and $\mathbf{6 h}$ exhibited two-fold anticandidal activity compared with ketoconazole against $C$. parapsilosis.

\section{Conflict of Interests}

The authors report no conflict of interests.

\section{Acknowledgments}

The study was supported by the Anadolu University Scientific Research Project, Eskişehir, Turkey (Project no. 1505S407). The authors present their thanks to Anadolu University Medicinal Plants, Drugs and Scientific Research Center (AÜBİBAM) for NMR analyses. The assistance of the staff is gratefully appreciated.

\section{References}

[1] N. C. Desai, N. Bhatt, H. Somani, and A. Trivedi, "Synthesis, antimicrobial and cytotoxic activities of some novel thiazole clubbed 1,3,4-oxadiazoles," European Journal of Medicinal Chemistry, vol. 67, pp. 54-59, 2013.

[2] S. Bondock, T. Naser, and Y. A. Ammar, "Synthesis of some new 2-(3-pyridyl)-4,5-disubstituted thiazoles as potent antimicrobial agents," European Journal of Medicinal Chemistry, vol. 62, pp. 270-279, 2013.

[3] N. M. Parekh, K. V. Juddhawala, and B. M. Rawal, "Antimicrobial activity of thiazolyl benzenesulfonamide-condensed 2,4thiazolidinediones derivatives," Medicinal Chemistry Research, vol. 22, no. 6, pp. 2737-2745, 2013.

[4] T. E. Glotova, M. Y. Dvorko, A. I. Albanov, O. N. Kazheva, G. V. Shilov, and O. A. D’yachenko, "1,3-Dipolar cycloaddition of 3-phenylamino-5-phenylimino-1,2,4-dithiazole to 1-acyl-2phenylacetylenes - a new route to functionalized 1,3-thiazole derivatives," Russian Journal of Organic Chemistry, vol. 44, no. 10, pp. 1532-1537, 2008.

[5] R. K. Yadlapalli, O. P. Chourasia, M. P. Jogi, A. R. Podile, and R. S. Perali, "Design, synthesis and in vitro antimicrobial activity of novel phenylbenzamido-aminothiazole-based azasterol mimics," Medicinal Chemistry Research, vol. 22, no. 6, pp. 2975-2983, 2013.

[6] M. A. Raslan and M. A. Khalil, "Heterocyclic synthesis containing bridgehead nitrogen atom: synthesis of 3-[(2H)2-oxobenzo[b]pyran-3-yl]-s-triazolo[3,4-b]-1,3,4-thiadiazine and thiazole derivatives," Heteroatom Chemistry, vol. 14, no. 2, pp. 114-120, 2003.

[7] J. Banothu, K. Vaarla, R. Bavantula, and P. A. Crooks, "Sodium fluoride as an efficient catalyst for the synthesis of 2,4disubstituted-1,3-thiazoles and selenazoles at ambient temperature," Chinese Chemical Letters, vol. 25, no. 1, pp. 172-175, 2014.

[8] K. A. Milinkevich, L. Ye, and M. J. Kurth, "Synthesis of 5-(thiazol-5-yl)-4,5-dihydroisoxazoles from 3-chloropentane2,4-dione," Journal of Combinatorial Chemistry, vol. 10, no. 4, pp. 521-525, 2008.

[9] M. A. Gouda, M. A. Berghot, G. E. Abd El-Ghani, and A. M. Khalil, "Synthesis and antimicrobial activities of some new thiazole and pyrazole derivatives based on 4,5,6,7tetrahydrobenzothiophene moiety," European Journal of Medicinal Chemistry, vol. 45, no. 4, pp. 1338-1345, 2010.

[10] P. C. Lv, K. R. Wang, Y. Yang et al., "Design, synthesis and biological evaluation of novel thiazole derivatives as potent FabH inhibitors," Bioorganic and Medicinal Chemistry Letters, vol. 19, no. 23, pp. 6750-6754, 2009. 
[11] A. Zablotskaya, I. Segal, A. Geronikaki et al., "Synthesis, physicochemical characterization, cytotoxicity, antimicrobial, antiinflammatory and psychotropic activity of new $\mathrm{N}-[1,3-$ (benzo)thiazol-2-yl]- $\omega$-[3,4-dihydroisoquinolin-2(1H)-yl]alkanamides," European Journal of Medicinal Chemistry, vol. 70, no. 23, pp. 846-856, 2013.

[12] C. N. Kavitha, J. P. Jasinski, B. J. Anderson, H. S. Yathirajan, and M. Kaur, "1-[4-(4-Hydroxyphenyl)piperazin-1-yl]ethanone," Acta Crystallographica Section E: Structure Reports Online, vol. 69, article o1671, 2013.

[13] C. N. Kavitha, J. P. Jasinski, B. J. Anderson, H. S. Yathirajan, and M. Kaur, "4-Methyl-N-(4-nitrobenzylidene)piperazin-1amine," Acta Crystallographica Section E: Structure Reports Online, vol. 69, no. 11, pp. o1687-o1688, 2013.

[14] L. Yurttaş, Z. A. Kaplancikli, and Y. Özkay, "Design, synthesis and evaluation of new thiazole-piperazines as acetylcholinesterase inhibitors," Journal of Enzyme Inhibition and Medicinal Chemistry, vol. 28, no. 5, pp. 1040-1047, 2013.

[15] CLSI, Methods for Dilution Antimicrobial Susceptibility Tests for Bacteria That Grow Aerobically Approved Standard, CLSI Document M7-A7, 7th edition, 2006.

[16] N. S. L. Perry, P. J. Houghton, A. E. Theobald, P. Jenner, and E. K. Perry, "In-vitro inhibition of human erythrocyte acetylcholine esterase by Salvia lavandulae folia essential oil and constituent terpenes," Journal of Pharmacy and Pharmacology, vol. 52, no. 7, pp. 895-902, 2000.

[17] G. L. Ellman, K. D. Courtney, V. Andres Jr., and R. M. Feather-Stone, "A new and rapid colorimetric determination of acetylcholinesterase activity," Biochemical Pharmacology, vol. 7, no. 2, pp. 88-95, 1961. 

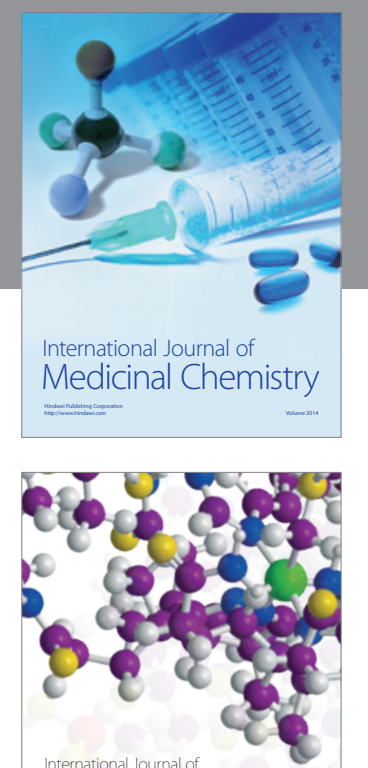

\section{Carbohydrate} Chemistry

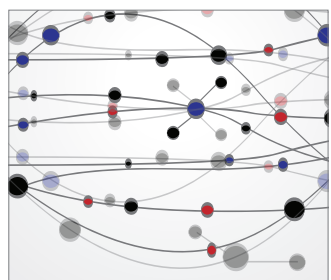

The Scientific World Journal
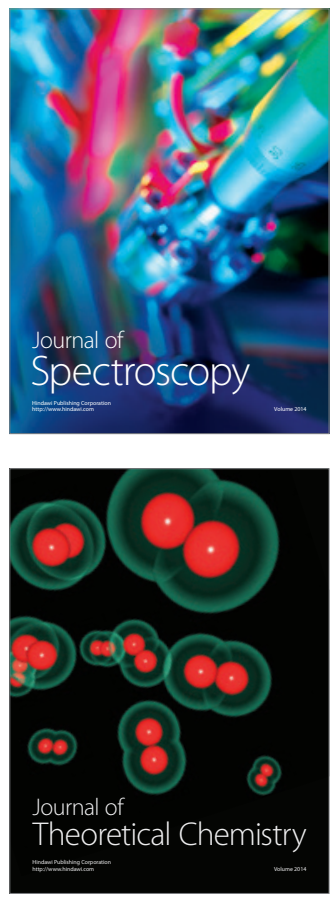
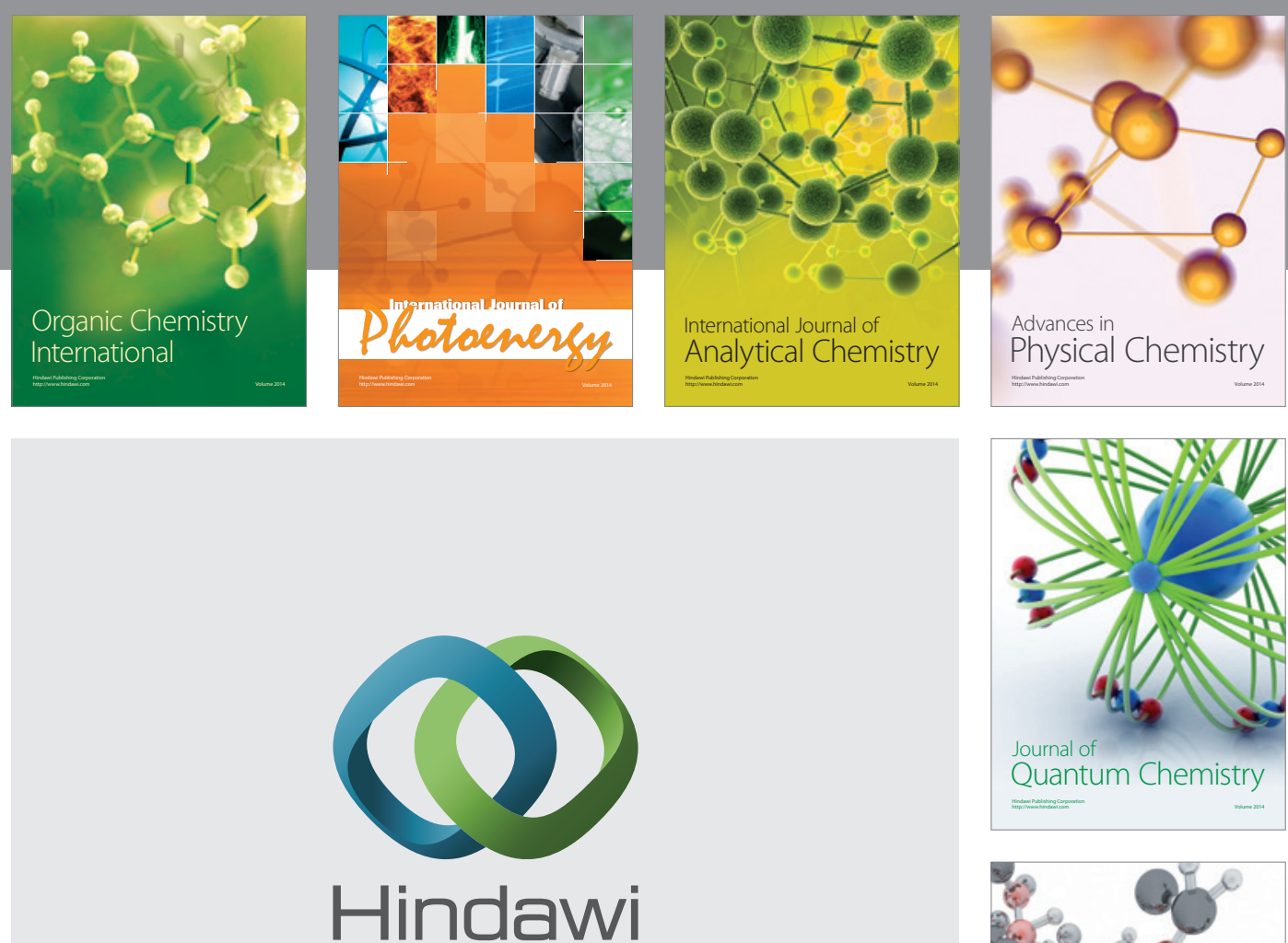

Submit your manuscripts at

http://www.hindawi.com

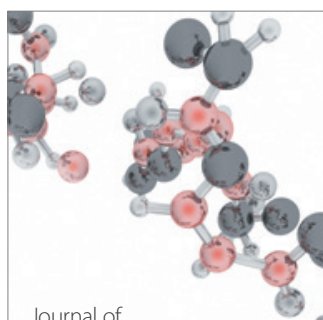

Analytical Methods

in Chemistry

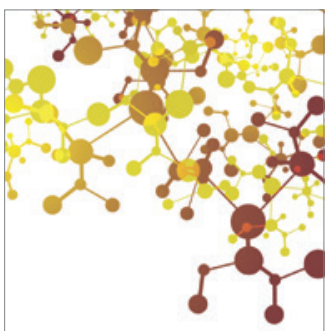

Journal of

Applied Chemistry

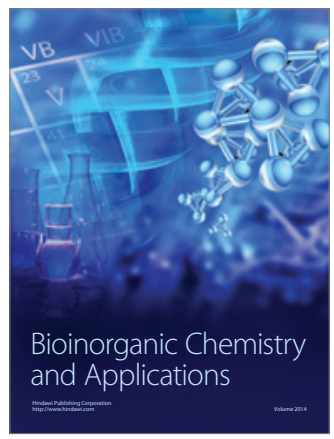

Inorganic Chemistry
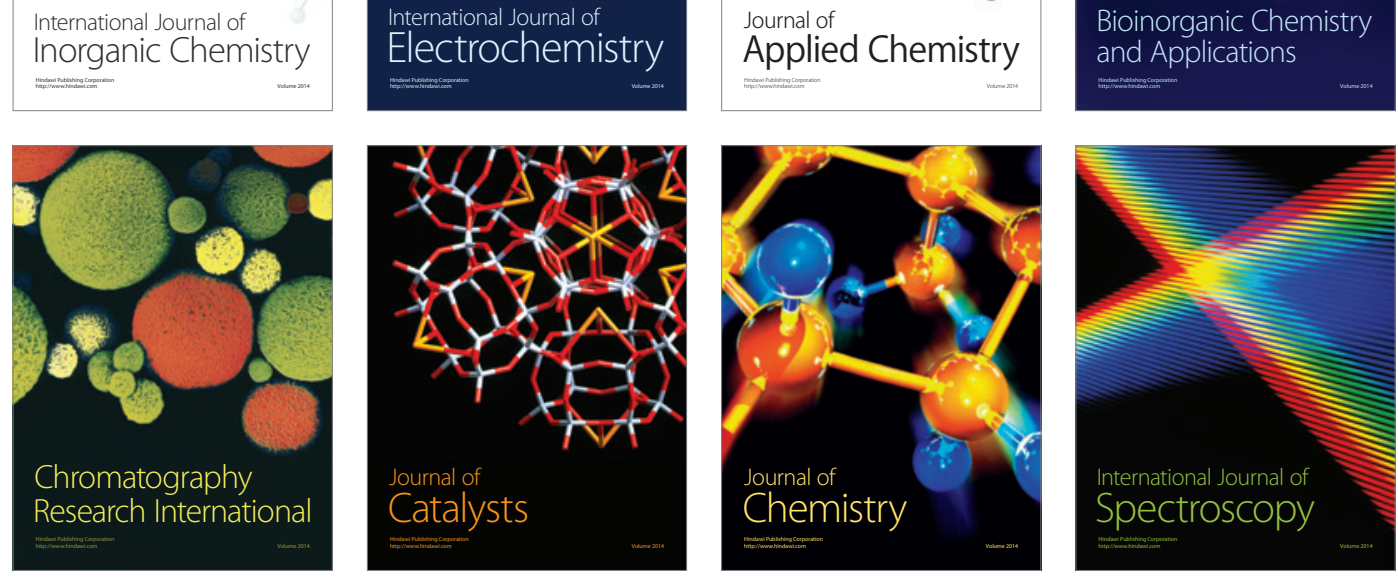